\title{
New ways with gravity waves
}

\section{There is a chance that the United States will have a detector capable of recognizing gravity waves in the next few years. But the federal budget deficit may be a hindrance.}

\begin{abstract}
The California and Massachusetts institutes of technology (Caltech and MIT respectively) are traditionally proud of their differences, sometimes recognizable as rivalry, but they have for some months been breaking new ground by working together on a joint application to the US National Science Foundation (NSF) for what will be the next gravity-wave detector most likely to succeed. The timetable is leisured, although that is not how it seems to those involved. They expect to have the financial gist of the application ready by next June, in time for the US budget cycle, and a more detailed specification of the equipment by September.
\end{abstract}

If President George Bush, the US Congress and the federal government's budget deficit allow, the budget for the fiscal year 1991 (beginning on 1 October 1990) may include an appropriation of $\$ 150$ million for a pair of detectors at opposite ends of the country (the Mohave desert and in Maine). There is talk of improving on that state of affairs with a third instrument elsewhere, Europe perhaps.

Each of the two institutions has had a vivid interest in the field since the early 1970s, when Dr Joseph Weber at the University of Maryland first demonstrated that gravity-wave detectors can be built and - less deliberately - how easily instrumental and spurious noise can be mistaken for genuine signals. Weber, it may be recalled, ran separate instruments on the campus of the University of Maryland, north of Washington DC, and at the Argonne National Laboratory, using a radio-link to compare the two signals (essential if local disturbances are to be eliminated). He explained at the time that he would have recorded the signals separately on magnetic tape if he had had the funds to pay for the stock of tape needed for a more objective comparison, much in the way that radioastronomy observatories collate measurements at separate telescopes. But the radio link could just as well have been the source of the coincidences that misled him.

It is interesting how solidly informed opinion has moved away from the instrument designs of a decade ago. Then, in the years after Weber's work, detectors were simply large symmetrical massive objects - aluminium cylinders, for example suspended so as to pick up mechanical movements from the surroundings. Earthquakes but also passing traffic are a nuisance. The crucial measurement is that of the displacement of the free surface of the suspended object, usually done by cementing a mirror to the surface and setting up an interferometer between that and an external source of light, naturally a laser.

The most obvious reason why detectors along these lines are out of fashion is mechanical. The sensitivity of a suspended object used for the detection of gravitational waves increases with its linear dimensions, but that complicates vibration-free suspension. Then there are unavoidable difficulties about the frequency spectrum of the detector, which is a complicated function of its geometry. In all the circumstances, it is remarkable that the sensitivity of the vibrating bars, which has steadily improved since Weber's first experiments (he is still active in the field), amounts to a relative strain of $10^{-18}$. The enthusiasts look for further improvements, perhaps by a total factor of 100 , but it seems to be agreed that there will then be a halt to the process, perhaps set by quantum noise (for which reason new designs of vibrating detectors are cooled to liquid helium temperature).

Luckily, it seems now to be agreed that there is a way forward to the sensitivity that may pick up gravitational waves from the distant objects in the Universe advertised to emit them with sensitivity in the range of $10^{-22}$ - colliding black holes and, more likely, exploding supernovae.

Logic (rather than NSF, which was unkindly reported by the Los Angeles Times last month to have decreed a "shotgun marriage") seems to have forced Caltech and MIT in similar directions. Each team has settled on the principle of building a laser interferometer from light traversing two perpendicular arms of an optical arrangement a little like that of a Michelson interferometer - a beam-splitter at the centre, directing light in two perpendicular directions, reflecting mirrors at the ends of the two arms and a light intensity meter for measuring phase differences when the two beams have been recombined by the beam splitter.

It is just about ten years that Ronald W. P. Drever, still very much a Scot, was explaining all this in a cramped (and not particularly clean) basement in the physics department of the University of Glasgow. He had then built one leg of an interferometer and was hoping to persuade the British grant agency, the Science and Engineering Research Council, to let him have a second, longer, leg. Now at Caltech, he is a proud acolyte of Caltech's twolegged instrument, each leg of which is $40-\mathrm{m}$ long. The dream is to scale up by a factor of 100, making each arm 4-km long.

Originally, Caltech and MIT were developing two different optical systems. But for the time being at least, they have combined forces. The sensitivity of an interferometer of the kind being planned in the measurement of the length of the respective legs of the equipment is evidently a direct function of the distance travelled, whence the quest for longer legs. But the same effect can be achieved by making the same light beam traverse each leg repeatedly, provided that the total time for which light is resident there does not exceed that in which there are significant variations of the forces due to gravitational waves.

At Caltech, the existing equipment has been built as if each leg were a FabryPerot resonant cavity, which is the agreed basis of the design that will be put forward next year. The idea is to stop the far end of each tunnel with a fully reflecting mirror, and the near end with a nearly matching partly transparent mirror. MIT's design, on ice for the time being, hangs on a trick in geometrical optics whereby rays enter each leg through an off-centre hole in an enclosing mirror, and are reflected repeatedly between one end of each leg and the other until, after a predictable number of double journeys, they reemerge through the aperture. In a 4-km leg, even 10,000 internal reflections would occupy only about a second.

The immediate goal is to build the pair of $4-\mathrm{km}$ instruments at a sensitivity of $10^{-20}$, whereupon two things should be possible: a realistic test of some of the predictions of the kinds of astrophysical phenomena generating detectable gravity waves, and the continued improvement of sensitivity. The obvious difficulty is that improvements are more difficult as the sensitivity is increased. It may be a long time before NSF has to finance a still more ambitious venture. Indeed, the next step will preferably carry the project outside the United States. With only two detectors, unambiguous measures of direction will not be possible. But with a third station, in Europe perhaps, triangulation would be feasible, so that the direction of radiating objects in the sky could be guessed at. That would enormously enhance the project.
John Maddox 